\title{
COMMENT
}

\section{Harmony and heresy of an L-type calcium channel inhibitor: suppression of cocaine seeking via increased dopamine transmission in the nucleus accumbens}

\author{
Sarah E. Swinford-Jackson ${ }^{1}$ and R. Christopher Pierce ${ }^{1}$ \\ Neuropsychopharmacology (2018) 43:2335-2336; https://doi.org/10.1038/s41386-018-0116-7
}

Cocaine addiction exacts devastating personal, societal, and economic costs. However, despite decades of research and numerous clinical trials, FDA approved treatments for cocaine use disorder are nonexistent. Given these failures, a potentially efficient strategy for identifying effective treatments for cocaine addiction is to repurpose pharmacotherapies that have already been FDA approved for other indications. This approach is particularly advantageous when existing preclinical and/or clinical research supports the mechanism of the FDA approved drug as a modulator of cocaine-related behaviors. One example is drugs targeting L-type calcium channels (LTCC), which have been implicated in a number of neuropsychiatric disorders including drug addiction [1]. In rodent models, blockade of LTCCs has been shown to attenuate cocaine reinforcement [2] as well as the reinstatement of cocaine seeking [3], an animal model of relapse.

In this issue of Neuropsychopharmacology, Addy et al. [4] explored the LTCC inhibitor isradipine (DynaCirc, Prescal), which is FDA approved for the treatment of hypertension, as a potential modulator of relapse-like behavior. In their model, rats were trained to self-administer cocaine and evaluated for cocaine cueevoked drug seeking following 10 days of forced abstinence. Systemic or intra-ventral tegmental area (VTA) administration of isradipine attenuated cocaine reinstatement. The current work also showed, importantly, that intra-VTA isradipine did not alter sucrose seeking, suggesting that the ability of this drug to suppress cocaine seeking is at least somewhat reinforcer specific and not due to general motor impairment. Interestingly, delivery of isradipine into the VTA immediately prior to a cocaine selfadministration session did not alter cocaine intake. To interpret these preclinical data in terms of translational potential, the LTCC inhibitor isradipine may help prevent cocaine relapse but apparently would not be efficacious as a cessation aid during periods of active drug taking.

The mechanism(s) by which isradipine modulates cocaine seeking are not known, but one prominent mediator of cueelicited cocaine seeking is dopamine signaling. A substantial literature indicates that increased dopamine transmission in the nucleus accumbens promotes cocaine seeking [5] and LTCC inhibitors, either directly or indirectly, suppress dopamine transmission [6, 7]. Addy et al. [4] used fast scan cyclic voltammetry to measure phasic and tonic dopamine release in the nucleus accumbens of rats that experienced identical selfadministration training and forced abstinence as those evaluated for cocaine- or sucrose seeking behavior. Surprisingly, intra-VTA administration of the same dose of isradipine that suppressed cocaine seeking actually increased phasic dopamine release relative to vehicle-treated rats. That is, in striking contradiction to cocaine reinstatement dogma, isradipine appears to attenuate cocaine seeking by enhancing phasic dopamine release in the accumbens. Tonic dopamine release in the nucleus accumbens also was higher after intra-VTA infusion of isradipine, even when factoring in the decreased tonic dopamine release induced by isradipine's ethanol vehicle. Systemic administration of isradipine recapitulated the increase in both tonic and phasic dopamine release in the accumbens, but it is unclear whether this effect was solely mediated by LTCCs in the VTA or may have been modulated by LTCCs localized to other regions. Nonetheless, Addy et al. [4] further showed that the effect of systemic isradipine on cocaine reinstatement was due to increased dopamine release in the nucleus accumbens because isradipine failed to alter cocaine seeking in rats that received intra-accumbens infusion of flupenthixol, a dopamine receptor antagonist. Flupenthixol alone dose-dependently attenuates cocaine seeking, so the authors chose a dose of flupenthixol that did not alter cocaine seeking relative to vehicle. The effectiveness of a sub-threshold dose of flupenthixol to block the suppression of cocaine seeking by isradipine, particularly when dopamine release is elevated, raises a number of interesting questions about the expression and signaling of dopamine receptors during cocaine abstinence.

An important caveat of these experiments is that dopamine release was measured in anesthetized rats; it will be essential to determine if the effects of isradipine on dopamine release remain consistent when assessed in awake, freely-moving rats and to measure dopamine release in the presence of cocaine-paired cues. Unlike many prior studies, which interrogated dopamine signaling in the context of reinstated drug seeking following extinction training, Addy et al. [4] assessed cocaine seeking following 10 days of forced abstinence. This is best characterized as an intermediate period of abstinence in which cue-evoked cocaine seeking is higher than immediately after cessation of cocaine-taking but is still not maximal, as seen at longer abstinence periods [8]. This well-described phenomenon, termed the incubation of cocaine craving, is known to be accompanied by myriad time-dependent changes in messenger RNA (mRNA) and protein expression [9], of which new potential mechanisms and/or targets are continuously being identified. It is conceivable that dopamine tone (not measured in this study) is modified during intermediate

${ }^{1}$ Center for Neurobiology and Behavior, Department of Psychiatry, Perelman School of Medicine at the University of Pennsylvania, Philadelphia, PA 19104, USA

Correspondence: R Christophe Pierce (rcpierce@pennmedicine.upenn.edu)

Received: 23 May 2018 Accepted: 31 May 2018

Published online: 9 June 2018 
abstinence from cocaine such that increased efflux is necessary to diminish cocaine seeking, though this will undoubtedly depend upon the status of countless other factors also influenced by abstinence from cocaine. Most prior studies have focused on early (i.e., 1-3 days) vs. late (i.e., 30-45 days) periods of abstinence; much less is known about molecular adaptations that occur between these timepoints. It will be critical to understand the mRNA and protein expression changes at both postsynaptic neurons in the accumbens, including dopamine receptors and the many protein partners with which they interact, as well as at the presynaptic side, including proteins that regulate dopamine release and, in particular, expression of LTCCs associated with intermediate abstinence from cocaine.

In this paper, Addy and colleagues make two important advances. First, they forcefully reiterate the point that LTCC inhibitors in general, and isradipine in particular, hold great promise as therapeutics for the treatment of cocaine relapse. Given the desperate need for medicines in this category, it is surprising that LTCC inhibitors have been only minimally assessed clinically as treatments for cocaine addiction [10]. Second, these authors make the highly novel observation that isradipine attenuates cocaine seeking at least in part by elevating dopamine release in the nucleus accumbens core. This finding is sure to engender debate and experimental reevaluation of the specific role of dopamine in cocaine cue-induce drug seeking. That said, dopamine is a rather mysterious modulator of neurotransmission that has been defying compartmentalization and specificity of function for decades. When it comes to dopamine, it seems that the more we know the less we know.

\section{ACKNOWLEDGEMENTS}

This work was supported by National Institutes of Health grants T32 DA28874 (SESJ), DA15214, and DA33641 (RCP).

\section{ADDITIONAL INFORMATION}

Competing interests: The authors declare no competing interests.

Publisher's note: Springer Nature remains neutral with regard to jurisdictional claims in published maps and institutional affiliations.

\section{REFERENCES}

1. Kabir ZD, Martinez-Rivera A, Rajadhyaksha AM. From gene to behavior: L-type calcium channel mechanisms underlying neuropsychiatric symptoms. Neurotherapeutics. 2017;14:588-613.

2. Kuzmin A, Zvartau E, Gessa GL, Martellotta MC, Fratta W. Calcium antagonists isradipine and nimodipine suppress cocaine and morphine intravenous selfadministration in drug-naive mice. Pharmacol Biochem Behav. 1992:41:497-500.

3. Anderson SM, Famous KR, Sadri-Vakili G, Kumaresan V, Schmidt HD, Bass CE, Terwilliger EF, Cha JH, Pierce RC. CaMKII: A biochemical bridge linking accumbens dopamine and glutamate systems in cocaine seeking. Nat Neurosci. 2008;11:344-53.

4. Addy NA, Nunes EJ, Hughley SM, Small KM, Baracz SJ, Haight JL, Rajadhyaksha AM. The L-type calcium channel blocker, isradipine, attenuates cue-induced cocaine-seeking by enhancing dopaminergic activity in the ventral tegmental area to nucleus accumbens pathway. Neuropsychopharmacology 2018, https:// doi.org/10.1038/s41386-018-0080-2, (e-pub ahead of print).

5. Willuhn I, Wanat MJ, Clark JJ, Phillips PE. Dopamine signaling in the nucleus accumbens of animals self-administering drugs of abuse. Curr Top Behav Neurosci. 2010;3:29-71.

6. Bonci A, Grillner P, Mercuri NB, Bernardi G. L-type calcium channels mediate a slow excitatory synaptic transmission in rat midbrain dopaminergic neurons. J Neurosci. 1998;18:6693-703.

7. Liu Y, Harding M, Pittman A, Dore J, Striessnig J, Rajadhyaksha A, Chen X. Cav1.2 and Cav1.3 L-type calcium channels regulate dopaminergic firing activity in the mouse ventral tegmental area. J Neurophysiol. 2014;112:1119-30.

8. Grimm JW, Hope BT, Wise RA, Shaham Y. Neuroadaptation. Incubation cocaine craving withdrawal. Nature. 2001;412:141-2.

9. Pickens $C L$, Airavaara M, Theberge F, Fanous S, Hope BT, Shaham Y. Neurobiology of the incubation of drug craving. Trends Neurosci. 2011;34:411-20.

10. Roache JD, Johnson BA, Ait-Daoud N, Mauldin JB, Thornton JE, Wells LT, Murff WL. Effects of repeated-dose isradipine on the abuse liability of cocaine. Exp Clin Psychopharmacol. 2005;13:319-26. 